\title{
Circulating microRNA profile in patients with membranous obstruction of the inferior vena cava
}

\author{
GUI-XIANG SUN ${ }^{1 *}$, YONG SU$^{1 *}$, YING LI $^{1 *}$, YA-FENG ZHANG ${ }^{1}$, LI-CHUN XU ${ }^{1}$, \\ MAO-HENG ZU ${ }^{2}$, SHUI-PING HUANG ${ }^{1}$, JIN-PENG ZHANG ${ }^{1}$ and ZHAO-JUN LU ${ }^{1}$ \\ ${ }^{1}$ Department of Public Health, Xuzhou Medical College; ${ }^{2}$ Department of Interventional Radiology, \\ Affiliated Hospital of Xuzhou Medical College, Xuzhou, Jiangsu 221004, P.R. China
}

Received October 19, 2014; Accepted November 3, 2015

DOI: $10.3892 / \mathrm{etm} .2016 .2981$

\begin{abstract}
Membranous obstruction of the inferior vena cava (MOVC) is a common type of Budd-Chiari syndrome. However, the pathogenesis of MOVC has not been fully elucidated. Recent studies demonstrated that microRNAs (miRNAs or miRs) are involved in multiple diseases. To the best of our knowledge, specific changes in the expression of miRNAs in MOVC patients have not been previously assessed. The present study used a microarray analysis, followed by reverse transcription-quantitative polymerase chain reaction (RT-qPCR) validation, with the aim to access the miRNA expression levels in the plasma of $34 \mathrm{MOVC}$ patients, compared with those in healthy controls. The results revealed a total of 16 differentially expressed miRNAs in MOVC patients. Subsequently, RT-qPCR analysis verified the statistically consistent expression of 5 selected miRNAs (miR-125a-5p, miR-133b, miR-423-5p, miR-1228-5p and miR-1266), in line with the results of the microarray analysis. These 5 miRNAs, which were described as crucial regulators in numerous biological processes and vascular diseases, may play an important role in the pathogenesis of MOVC. Bioinformatics analysis of target genes of the differentially expressed miRNAs revealed that these predicted targets were significantly enriched and involved in several key signaling pathways important for MOVC, including the ErbB, Wnt, MAPK and VEGF signaling pathway. In conclusion, miRNAs may involve in multiple signaling pathways contributing to the pathological processes of MOVC. The present study offers an intriguing new perspective on the involvement of miRNAs in
\end{abstract}

Correspondence to: Professor Zhao-Jun Lu, Department of Public Health, Xuzhou Medical College, 209 Tongshan Road, Xuzhou, Jiangsu 221004, P.R. China

E-mail: lu23zj@126.com

*Contributed equally

Key words: microRNA, Budd-Chiari syndrome, membranous obstruction of the inferior vena cava, bioinformatics analysis
MOVC; however, the precise underlying mechanisms require further validation.

\section{Introduction}

Budd-Chiari syndrome (BCS) is a heterogeneous disorder that occurs due to an obstruction present between the hepatic venules and the junction of the inferior vena cava, resulting in significant mortality and morbidity (1). It is a complex disease with a wide spectrum of etiologies and presentations (2). BCS remains rare with incidence rates of $0.2-0.8$ million people affected per year $(3,4)$

Numerous studies have suggested that the clinical features and etiology distribution of BCS vary according to the geographical area (5-7). In western countries, pure hepatic venule obstruction accounts for $>50 \%$ of BCS cases, whereas thrombosis is the predominant pathological lesion of BCS (1). The majority of cases are closely associated with underlying inherited or acquired thrombotic risk factors, with $\sim 80 \%$ of BCS patients presenting at least one thrombotic risk factor (8). Myeloproliferative neoplasms (MPNs) are considered to be the leading cause in the development of BCS, and have been reported in $\sim 50 \%$ of BCS patients (9). In developing countries, including Nepal and China, the most common type of $\mathrm{BCS}$ is membranous obstruction of the inferior vena cava (MOVC) $(7,10)$, which has been shown to account for $62.7 \%$ of BCS cases in China (11). Recent data from several centers have consistently shown that underlying thrombotic disorders in Chinese BCS patients are rarely detected (6). In addition, certain risk factors that have been confirmed in western countries, such as MPNs, do not seem to be etiological factors in Chinese BCS patients $(12,13)$. This observation suggests that the etiological distribution of BCS may differ between western countries and China. Accordingly, due to the potentially different pathogenesis factors in BCS patients of different ethnicities (14), the present study focused on Chinese patients with BCS.

MicroRNAs (miRNAs or miR) are a class of naturally small noncoding RNAs with a length of 21-23 nucleotides, which bind to the 3'-untranslated regions of their target mRNAs and regulate gene expression at the post-transcriptional level (15). Previous studies have revealed that miRNAs serve a crucial role in numerous pathological and physiological processes, 
including organ development, cell differentiation, immune function and vascular diseases $(16,17)$. In addition, a number of miRNAs have been identified as important modulators of vascular pathologies, including apoptosis, angiogenesis, inflammation, hypertension and atherosclerosis (18). Furthermore, the genetic abnormality or dysregulation of miRNAs may contribute to the pathogenesis of vascular diseases $(19,20)$; thus, these findings demonstrate that miRNAs play an important role in vascular disorders.

Certain studies have demonstrated that the amount of circulating miRNAs may be used as clinical biomarkers $(21,22)$. However, to the best of our knowledge, cell-free miRNAs in the plasma of BCS patients with MOVC have not been previously investigated. Therefore, the aim of the present study was to identify a panel of plasma miRNAs that are differentially expressed in patients with MOVC and to investigate the potential biological function of these candidate miRNAs.

\section{Materials and methods}

Study population. The study was approved by the Ethics Committee of the Affiliated Hospital of Xuzhou Medical College (Xuzhou, China), and informed consent was obtained from each subject.

A total of 34 patients aged 27-75 years who were diagnosed with incident MOVC at the Affiliated Hospital of Xuzhou Medical College between February 2013 and September 2013 were enrolled into the present study. Patients were diagnosed based on the BCS characteristics, as previously described (REFold1). All diagnoses were confirmed using radiographic imaging, including Doppler ultrasound and magnetic resonance imaging. In addition, venography images suggested the existence of a membrane in the inferior vena cava. Patients with other coexisting diseases, including hypertension, coronary heart disease, diabetes, blood diseases and cancer, were excluded. Additionally, 30 healthy age- and gender-matched subjects were recruited as the controls. The microarray cohort of subjects included 9 MOVC patients and 5 healthy volunteers (MOVC-1/controls-1). The present study also investigated a second group composed of 25 MOVC patients and 25 healthy controls (MOVC-2/controls-2) for independent validation using reverse transcription-quantitative reverse transcription (RT-qPCR) analysis. No statistically significant differences were detected in the age and gender distribution of patients and controls (Table I).

Plasma collection and RNA isolation. A 5-ml sample of venous blood was collected from each patient or healthy volunteer before breakfast on the morning after hospital admission. Blood samples were drawn into EDTA-containing tubes and the plasma was immediately separated by a two-step centrifugation protocol at room temperature (centrifugation at $1,800 \mathrm{x} \mathrm{g}$ for $10 \mathrm{~min}$, followed by centrifugation at $15,000 \mathrm{x} \mathrm{g}$ for $10 \mathrm{~min}$ ) (23). The supernatant was then transferred into RNase-free microcentrifuge tubes and stored at $-80^{\circ} \mathrm{C}$ until use.

Total plasma RNA was harvested with the RNeasy mini kit (Qiagen, Hilden, Germany) according to the manufacturer's instructions. Briefly, $200 \mu \mathrm{l}$ plasma was diluted with $1 \mathrm{ml}$ QIAzol lysis reagent. After a 5-min incubation, $5 \mu \mathrm{l}$ of
5 nmol/l synthetic Caenorhabditis elegans miR-39 (Shanghai GenePharma Co., Ltd., Shanghai, China) was added to each sample as a spike control (24). Next, phenol extraction and various filter cartridge steps were performed according to the manufacturer's instructions. A NanoDrop 2000 spectrophotometer (NanoDrop Technologies; Thermo Fisher Scientific Inc., Wilmington, DE, USA) was used to measure the quality and concentration of RNA in each plasma sample.

miRNAs expression profiling. In order to assess the levels of specific miRNAs in the patients with MOVC, miRNA expression profiling of the plasma samples from 9 MOVC-1 patients and 5 healthy control-1 participants was performed using miRNA microarray analysis. As BCS is a rare disease, the maximum number of patients with MOVC were selected and the number of controls were reduced accordingly due to economic constraints. Microarray analysis and RT-qPCR are two common miRNA detection methods (25). In order to detect the miRNA expression levels of the samples, high-throughput microarray experiments were performed by KangChen Bio-tech Inc. (Shanghai, China), as this technique is highly sensitive and time-efficient. However, as the detection results may contain certain errors, it was necessary to conduct further validation using RT-qPCR, as this technique is considered the gold standard for detecting miRNAs (26). Therefore, independent expanding samples were chosen for RT-qPCR validation in order to make the results more reliable, as through microarray analysis and subsequent RT-qPCR validation the dysregulated miRNAs in patients with MOVC can be reflected with increased accuracy.

Briefly, $3 \mu \mathrm{g}$ RNA samples were labeled with the Exiqon Hy3/Hy5 power labeling kit (Exiqon, Vedbaek, Denmark) and hybridized on the miRCURY LNA ${ }^{\mathrm{TM}}$ array (version 18.0; Exiqon), which contains 3,100 capture probes, covering all human miRNAs annotated in the miRBase database (http://www.mirbase.org/). Subsequently, the slides were scanned using a GenePix 4000B microarray laser scanner (Axon Instruments; Molecular Devices, LLC, Sunnyvale, CA, USA) and microarray images were analyzed using GenePix Pro 6.0 software. The green signal intensity was calculated following background subtraction, and the average of replicated spots on the same slide was calculated to determine the median intensity. The median normalization method was used to acquire normalized data (foreground - background / median). The threshold value for statistical significance used to define upregulated or downregulated miRNAs was a fold change of $>2$, with a value of $\mathrm{P}<0.05$ calculated by the Student's $t$ test.

RT-qPCR platform for relative quantification of miRNAs. In order to confirm the miRNA array results, stem-loop RT-qPCR was performed (Table II). Briefly, $5 \mu 1$ total RNA was reverse-transcribed into cDNA in a volume of $15 \mu \mathrm{l}$ with the TaqMan MicroRNAs Reverse Transcription kit (Applied Biosystems; Thermo Fisher Scientific Inc., Carlsbad, CA, USA). Subsequently, PCR was performed in $15 \mu$ l reaction mixtures using the GeneAmp PCR system 9700 (Applied Biosystems; Thermo Fisher Scientific Inc.). The samples were subjected to thermal cycling parameters of $30 \mathrm{~min}$ at $16^{\circ} \mathrm{C}$, $40 \mathrm{~min}$ at $42^{\circ} \mathrm{C}$, and $5 \mathrm{~min}$ at $85^{\circ} \mathrm{C}$, and then kept at $4^{\circ} \mathrm{C}$. qPCR reactions were performed in triplicate using the TaqMan PCR 
Table I. Characteristics of the study samples.

\begin{tabular}{|c|c|c|c|c|c|c|}
\hline \multirow[b]{2}{*}{ Characteristic } & \multicolumn{3}{|c|}{ miRNA microarray } & \multicolumn{3}{|c|}{ RT-qPCR } \\
\hline & $\operatorname{MOVC}(\mathrm{n}=9)$ & Control (n=5) & P-value & $\operatorname{MOVC}(n=25)$ & Control $(n=25)$ & P-value \\
\hline Male gender, n (\%) & $3(33)$ & $2(40)$ & 0.80 & $15(60)$ & $15(60)$ & 1.00 \\
\hline Age, years ${ }^{\mathrm{a}}$ & $42.6(27.5-60.3)$ & $42.5(28.1-1.7)$ & 0.65 & $48.1(27.3-74.6)$ & $48.0(26.9-74.8)$ & 0.98 \\
\hline
\end{tabular}

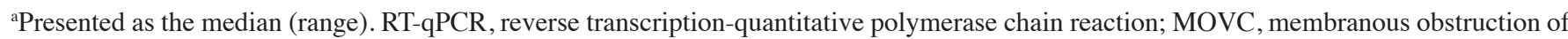
the inferior vena cava.

Table II. Characterization of miRNAs selected for reverse transcription-quantitative polymerase chain reaction validation.

\begin{tabular}{llr}
\hline miRNA & \multicolumn{1}{c}{ Primer sequence } & miRBase accession number \\
\hline hsa-miR-125a-5p & UCCCUGAGACCCUUUAACCUGUGA & MIMAT0000443 \\
hsa-miR-423-5p & UGAGGGGCAGAGAGCGAGACUUU & MIMAT0004748 \\
hsa-miR-133b & UUUGGUCCCCUUCAACCAGCUA & MIMAT0000770 \\
hsa-miR-1228-5p & GUGGGCGGGGGCAGGUGUGUG & MIMAT0005582 \\
hsa-miR-1266 & CCUCAGGGCUGUAGAACAGGGCU & MIMAT0005920
\end{tabular}

Table III. Differentially expressed miRNAs in MOVC-1 patients, as compared with healthy control-1 participants.

\begin{tabular}{lccc}
\hline miRNAs & Fold change & Regulation & P-value \\
\hline hsa-miR-423-5p & 4.22 & $\mathrm{Up}$ & 0.0032 \\
hsa-miR-133b & 3.42 & $\mathrm{Up}$ & 0.0012 \\
hsa-miR-125a-5p & 2.89 & $\mathrm{Up}$ & 0.0140 \\
hsa-miR-1299 & 2.71 & $\mathrm{Up}$ & 0.0099 \\
hsa-miR-1265 & 2.56 & $\mathrm{Up}$ & 0.0380 \\
hsa-miR-296-3p & 2.01 & $\mathrm{Up}$ & 0.0370 \\
hsa-miR-1266 & 0.09 & Down & 0.0040 \\
hsa-miR-1228-5p & 0.12 & Down & 0.0006 \\
hsa-miR-659-5p & 0.15 & Down & 0.0060 \\
hsa-miR-3133 & 0.22 & Down & 0.0002 \\
hsa-miR-523-3p & 0.32 & Down & 0.0065 \\
hsa-miR-301a-5p & 0.37 & Down & 0.0080 \\
hsa-miR-299-5p & 0.44 & Down & 0.0110 \\
hsa-miR-513a-5p & 0.45 & Down & 0.0280 \\
hsa-miR-149-3p & 0.45 & Down & 0.0240 \\
hsa-miR-337-3p & 0.47 & Down & 0.0030 \\
\hline
\end{tabular}

MOVC, membranous obstruction of the inferior vena cava.

Master Mix on an ABI 7500 system (Applied Biosystems; Thermo Fisher Scientific Inc.). Each amplification reaction was performed in a volume of $20 \mu \mathrm{l}$ containing $1.33 \mu \mathrm{l}$ cDNA and $1 \mu \mathrm{l}$ of gene-specific primers. The cycle threshold $(\mathrm{Cq})$ values were calculated with the SDS version 2.0.6 software (Applied Biosystems; Thermo Fisher Scientific Inc.), and the fold change for each miRNA was calculated using the comparative method $\left(2^{-\Delta \Delta \mathrm{Cq}}\right)$ with cel-miR-39 as the endogenous control (27).

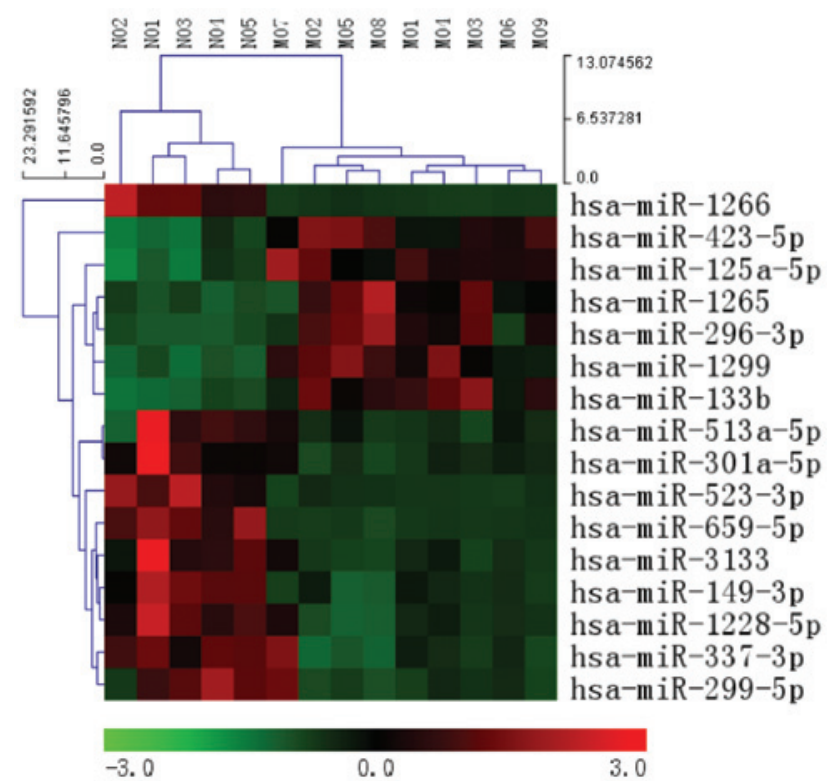

Figure 1. Heat map showing 16 differentially expressed miRNAs (fold change of $>2)$ in the plasma of MOVC-1 patients $(n=9)$ and healthy control-1 participants $(n=5)$. Each row represents one miRNA, and each column represents a plasma sample. The relative miRNA expression is depicted according to the color scale. Red indicates upregulation and green indicates downregulation. Denotations beginning with M indicate the MOVC patients, while those beginning with $\mathrm{N}$ indicate the healthy controls. MOVC, membranous obstruction of the inferior vena cava.

Prediction of target genes and bioinformatics analysis of gene functions. In order to predict the target genes of differentially expressed miRNAs that were significantly dysregulated in the plasma of MOVC patients, the online databases Miranda (http://www.microrna.org), miRDB (http://mirdb.org/miRDB/) and TargetScan (http://www.targetscan.org) were used. In the 
Table IV. KEGG pathway analysis of putative target genes regulated by differentially expressed miRNAs.

A, Upregulated miRNAs

\begin{tabular}{ll}
\hline Analysis for predicted target genes & P-value \\
\hline Pathways in cancer & $2.55 \times 10^{-10}$ \\
Axon guidance & $6.90 \times 10^{-8}$ \\
ErbB signaling pathway & $6.52 \times 10^{-6}$ \\
Insulin signaling pathway & $1.15 \times 10^{-5}$ \\
Focal adhesion & $3.41 \times 10^{-5}$ \\
Wnt signaling pathway & $1.15 \times 10^{-4}$ \\
MAPK signaling pathway & $1.30 \times 10^{-4}$ \\
VEGF signaling pathway & $3.39 \times 10^{-4}$ \\
p53 signaling pathway & $1.23 \times 10^{-3}$ \\
\hline
\end{tabular}

B, Downregulated miRNAs

\begin{tabular}{lc}
\hline Analysis for predicted target genes & P-value \\
\hline Pathways in cancer & $2.96 \times 10^{-9}$ \\
ErbB signaling pathway & $1.45 \times 10^{-6}$ \\
Wnt signaling pathway & $1.67 \times 10^{-5}$ \\
p53 signaling pathway & $2.93 \times 10^{-5}$ \\
T cell receptor signaling pathway & $5.47 \times 10^{-5}$ \\
Apoptosis & $1.70 \times 10^{-4}$ \\
Vascular smooth muscle contraction & $4.99 \times 10^{-4}$ \\
MAPK signaling pathway & $7.90 \times 10^{-4}$ \\
VEGF signaling pathway & $3.69 \times 10^{-3}$
\end{tabular}

KEGG, Kyoto Encyclopedia of Genes and Genomes; VEGF, vascular endothelial growth factor; MAPK, mitogen-activated protein kinase.

present study, a predicted gene was considered as a putative target candidate when it was predicted by at least two of the aforementioned databases.

The predicted miRNA target genes were analyzed for Gene Ontology (GO)annotation andKyotoEncyclopediaofGenesand Genomes(KEGG) pathway enrichmentusing the onlineDAVID Bioinformatics Resources (http://david.abcc.ncifcrf.gov/). Fisher's exact test and $\chi^{2}$ test were used to classify the GO category and the pathway analysis. Only GO annotations or pathways with a P-value of $<0.01$ and false discovery rate of $<0.05$ were selected.

Statistical analysis. SPSS version 16.0 software (SPSS, Inc., Chicago, IL, USA) was used to analyze the dataset from miRNA microarray experiments. Descriptive statistics were applied to determine differential individual characteristics between MOVC patients and healthy controls by the Mann-Whitney $U$ test for continuous variables and the $\chi^{2}$ test or Fisher exact test (two-tailed) for categorical variables. Statistical graph analysis was performed using GraphPad Prism 5.0 (GraphPad Software Inc., La Jolla, CA, USA). Differences with $\mathrm{P}<0.05$ (two-tailed) were considered to be statistically significant.
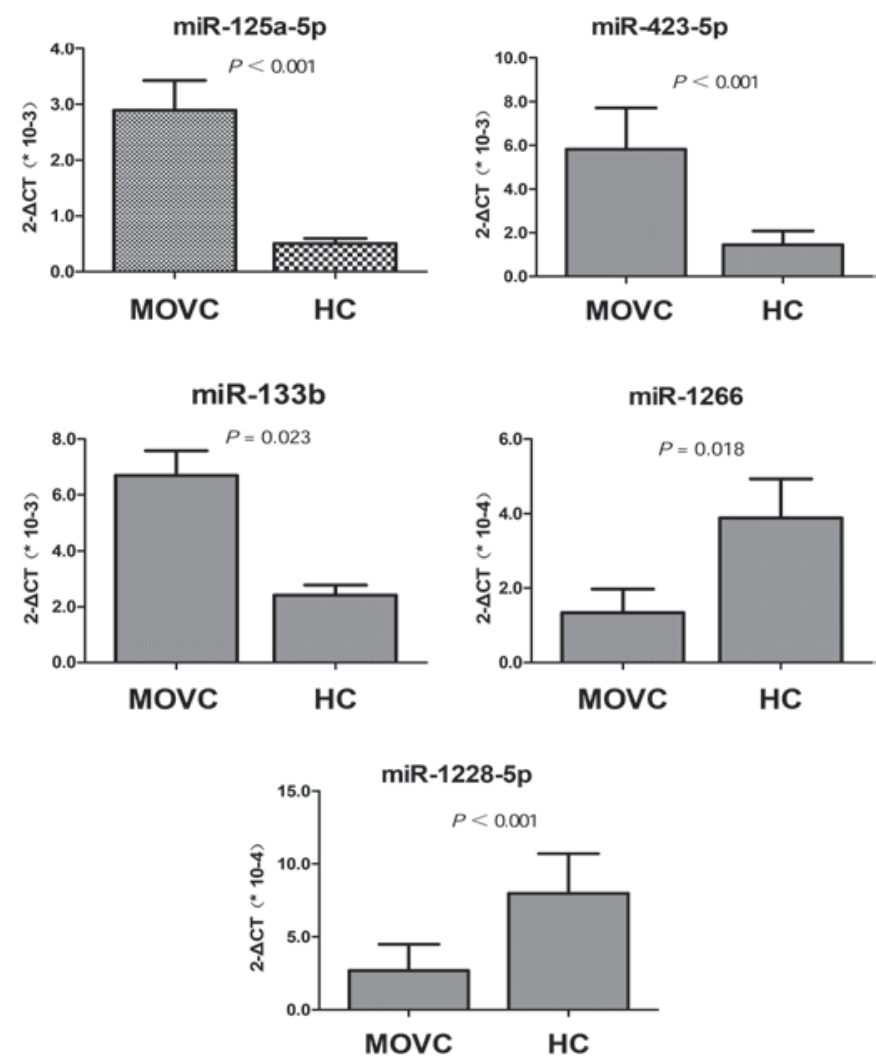

Figure 2. Circulating miRNAs in patients with MOVC-2, as compared with healthy control-2 participants. The expression of 5 selected miRNAs in EDTA-plasma obtained from patients with MOVC $(n=25)$ and $H C(n=25)$, were determined using reverse transcription-quantitative polymerase chain reaction. The relative expression of each miRNA in MOVC patients compared with the healthy control subjects was normalized to the expression of cel-miR-39. P-values were calculated by two-sided Student's t test. MOVC, membranous obstruction of the inferior vena cava; $\mathrm{HC}$, healthy control.

\section{Results}

MicroRNA microarray expression profiling. Following normalization of the raw data, a total of 16 microRNAs were found to be significantly differentially expressed in the MOVC-1 patients, as compared with the controls-1 ( $>2$-fold change; $\mathrm{P}<0.05)$. Among them, 6 upregulated miRNAs were identified, while 10 downregulated miRNAs were identified between the two groups (Table III). Unsupervised hierarchical clustering analysis was conducted using the expression levels of these 16 differentially expressed miRNAs, resulting in clear differentiation of MOVC samples from normal samples into two different clusters (Fig. 1).

Validation of profiling data using RT-qPCR. To verify the findings obtained through the miRNA profile analysis, RT-qPCR was performed in the independent cohort of 25 MOVC-2 patients and 25 healthy control-2 participants. The investigation was focused on 5 candidate miRNAs (miR-125a-5p, miR-133b, miR-423-5p, miR-1266 and miR-1228-5p), based on their differential expression levels observed in the present microarray analysis $(2.89,3.42,4.22,0.09$ and 0.12 -fold changes, respectively) (Table III) and their roles as crucial regulators of various biological processes and vascular diseases (28-32). The RT-qPCR results were consistent with 
A

Sig G0 terms of DE gene $(-\operatorname{Lg} P)$

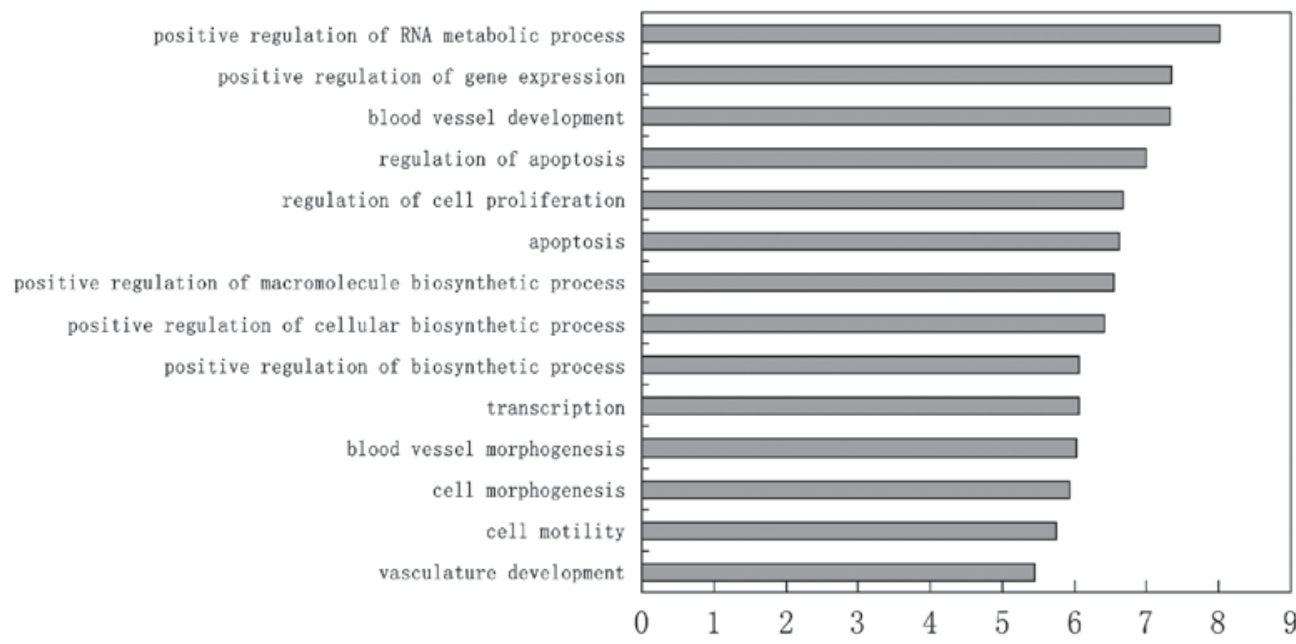

B Sig G0 terms of DE gene $(-\operatorname{Lg} P)$

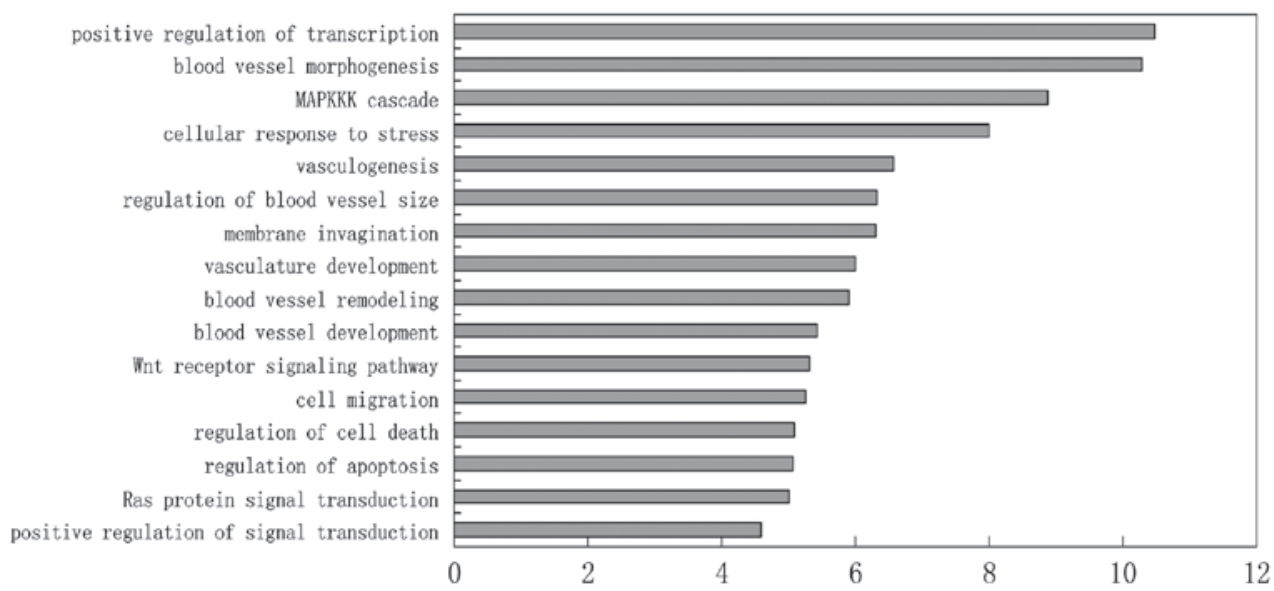

Figure 3. GO annotations for the predicted target genes. The charts compose the GO terms targeted by (A) upregulated and (B) downregulated miRNAs. The $\mathrm{y}$-axis shows the GO category and the $\mathrm{x}$-axis shows the enrichment of GOs. Sig, significance; GO, gene ontology; DE, differentially expressed; Lg $P_{2} \mathrm{Lg} P$-value; MAPKKK, mitogen-activated protein kinase kinase kinase.

the microarray data (Fig. 2). Specifically, the expression levels of miR-125a-5p, miR-133b and miR-423-5p were significantly increased, whereas those of miR-1228-5p and miR-1266 were significantly decreased in the MOVC patients. Therefore, the RT-qPCR results validated the observed microarray data, suggesting that the data obtained from the miRNA microarrays accurately reflected the miRNA expression levels.

Bioinformatics analysis of miRNAs. Three available databases (Miranda, miRBase and TargetScan) were used to predict targets genes of the 16 miRNAs that were differentially expressed in the MOVC-1 and control-1 groups. In order to reduce the possibility of a false positive, only targets estimated by at least two of these databases were considered as putative candidates. To assess the potential biological impact of the differentially expressed miRNAs, the present study performed GO annotations and KEGG pathway enrichment analysis for the predicted target genes. Employing that criterion, 20,009 target genes were predicted for the upregulated miRNAs, including Janus kinase 2 and vascular endothelial growth factor B (VEGFB); whereas 25,831 target genes were predicted for the downregulated miRNAs, including mitogen-activated protein kinase (MAPK) and patelet-derived growth factor. The primary GO terms targeted by upregulated miRNAs were the response to blood vessel development and morphogenesis, apoptosis and cell motility. By contrast, significant GO terms corresponding to downregulated miRNAs appeared to be the regulation of blood vessel morphogenesis, the MAPK kinase kinase cascade, blood vessel remodeling and Wnt receptor signaling pathway (Fig. 3).

KEGG pathway analysis showed that the target genes for upregulated miRNAs were associated with pathways in cancer, axon guidance, focal adhesion and the ErbB and Wnt signaling pathways. The target genes for downregulated miRNAs were associated with pathways in cancer, as well as with the ErbB, Wnt, p53 and T cell receptor signaling pathways. Notably, certain signaling pathways, such as pathways in cancer and the ErbB, Wnt, MAPK and VEGF signaling pathways, were targeted by the upregulated and downregulated miRNAs, suggesting that extensive miRNA regulation of these pathways is involved in MOVC (Table IV). 


\section{Discussion}

The levels of circulating miRNAs potentially reflect altered pathological and physiological conditions (33). As previously discussed, numerous studies have shown that miRNAs serve a crucial role in vascular disorders $(19,20)$. However, the gene regulation of miRNAs in the pathogenesis of MOVC has not been extensively investigated. Thus, the present study aimed to assess the miRNA expression profiles in the plasma of patients with MOVC in comparison with those in age- and gender-matched healthy individuals.

The profiling data in the present study demonstrated a distinct miRNA expression profile in MOVC patients, including 6 miRNAs that were significantly upregulated and 10 miRNAs that were significantly downregulated. Furthermore, 5 significantly altered miRNAs (miR-125a-5p, miR-133b, miR-423-5p, miR-1266 and miR-1228-5p) were verified in a second cohort of patients using RT-qPCR, and the results were found to be in line with those obtained by the miRNA microarray analysis, thus suggesting that the profiling results were reliable. Among the dysregulated miRNAs (Table III), miR-125a-5p has previously been reported to have a higher expression level in vascular endothelial cells (VECs) and brain endothelial cells $(34,35)$, and thus potentially contributing various vascular diseases, including atherosclerosis, hypertension and stroke. It has been reported that the upregulation of miR-125a-5p may be involved in angiogenesis defects in mature endothelial cells by targeting the associated transcriptional enhancer factor-1 (36). Additionally, Li et al (34) identified that miR-125a-5p was able to inhibit endothelin-1 (ET-1) expression in VECs, while Hao et al (37) found that miR-125a-5p was able to suppress the expression of ET-1 in the coronary arteries. ET-1, as a potent vasoconstrictive peptide, plays critical roles in the progression of atherosclerosis, vascular inflammation and remodeling $(38,39)$.

miR-133b, a myogenic miRNA, has been shown to have a close association with multiple tumors and is generally considered to have tumor-suppressive functions $(40,41)$. It is also overproduced in early myocardial injury subsequent to heart transplantation (42), suggesting its association with cardiovascular system disorders. Furthermore, miR-423-5p, which has been reported to be upregulated in human failing myocardium, may be a novel blood-based biomarker for heart failure (43). Thus far, studies concerning miR-1266 and miR-1228-5p are limited, with only a small number of reports investigating their role in cancer $(44,45)$. Combining the aforementioned findings and our results, these dysregulated miRNAs (miR-125a, miR-133b, miR-423-5p, miR-1266 and miR-1228-5p) are suggested to be closely associated with the pathogenesis of MOVC; however, the detailed underlying mechanism requires further investigation.

To further clarify the role of miRNAs in the pathogenesis of MOVC, GO annotations and KEGG pathway analysis were performed on the target genes known to be regulated by the differentially expressed miRNAs. KEGG pathway analysis showed significant changes associated with the ErbB, Wnt, MAPK and VEGF signaling pathways in the MOVC patients, as compared with the healthy controls. ErbB signaling is implicated in the regulation of the normal function of the embryonic and adult heart (46). The Wnt signaling pathway plays an important role in morphogenesis, cell survival, differentiation and proliferation (47). Additionally, the MAPK and VEGF signaling pathways are closely associated with the function of VECs. Siddiqui et al (48) found that MAPK had a considerable effect on the endothelial monolayer integrity by signaling proliferation and survival of endothelial cells. VEGF, which is crucial in angiogenesis, is an endothelial cell-specific mitogen (49) that regulates endothelial cell proliferation, migration, vascular permeability, secretion and other endothelial functions (50). Bioinformatics analysis performed in future studies may improve the understanding on the pathogenesis of MOVC.

To the best of our knowledge, the present study is the first to evaluate the plasma miRNA expression pattern in MOVC patients by microarray-based miRNA analysis. In conclusion, a total of 16 differentially expressed miRNAs were identified in the plasma between MOVC patients and healthy controls. In total, 5 of these miRNAs were verified by RT-qPCR, which provided results in line with those obtained by the miRNA microarray analysis. Functional bioinformatics analysis demonstrated that the target genes regulated by these miRNAs were involved in several biological processes and signaling pathways. The study of these miRNAs may provide a clearer understanding on the pathogenesis of MOVC and help identify novel methods for the diagnosis, treatment and prevention of MOVC. However, further investigation using more samples is required to verify the differential expression of the miRNAs observed in the present study, and their cellular origin and function in MOVC should be analyzed.

\section{Acknowledgements}

The present study was supported by grants from the Priority Academic Program Development of Jiangsu Higher Education Institutions (grant no. 2012SJB190015) and the National Natural Science Foundation of China (grant no. 81172604).

\section{References}

1. Valla DC: Primary Budd-Chiari syndrome. J Hepatol 50: 195-203, 2009.

2. Hefaiedh R, Cheikh M, Marsaoui L, Ennaifer R, Romdhane H, Ben Nejma H, Bel Hadj N, Arfa N and Khalfallah MT: The Budd-Chiari syndrome. Tunis Med 91: 376-381, 2013.

3. Leebeek FW, Smalberg JH and Janssen HL: Prothrombotic disorders in abdominal vein thrombosis. Neth J Med 70: 400-405, 2012.

4. Plessier A, Rautou PE and Valla DC: Management of hepatic vascular diseases. J Hepatol 56: S25-S38, 2012.

5. Plessier A and Valla DC: Budd-Chiari syndrome. Semin Liver Dis 28: 259-269, 2008.

6. Qi X, Han G, Wu F, Ren W, He C, Yin Z, Niu J, Bai M, Yang Z, Wu K and Fan D: Thrombotic risk factors in Chinese Budd-Chiari syndrome patients. An observational study with a systematic review of the literature. Thromb Haemost 109: 878-884, 2013.

7. Cheng D, Xu H, Lu ZJ, Hua R, Qiu H, Du H, Xu X and Zhang J: Clinical features and etiology of Budd-Chiari syndrome in Chinese patients: A single. center study. J Gastroenterol Hepatol 28: 1061-1067, 2013.

8. Darwish Murad S, Plessier A, Hernandez-Guerra M, Fabris F, Eapen CE, Bahr MJ, Trebicka J, Morard I, Lasser L, Heller J, et al; EN-Vie (European Network for Vascular Disorders of the Liver): Etiology, management and outcome of the Budd-Chiari syndrome. Ann Intern Med 151: 167-175, 2009.

9. Smalberg JH, Arends LR, Valla DC, Kiladjian JJ, Janssen HL and Leebeek FW: Myeloproliferative neoplasms in Budd-Chiari syndrome and portal vein thrombosis: A meta-analysis. Blood 120: 4921-4928, 2012. 
10. Okuda K: Membranous obstruction of the inferior vena cava (obliterative hepatocavopathy, Okuda). J Gastroenterol Hepatol 16: 1179-1183, 2001.

11. Wang ZG, Zhang FJ, Yi MQ and Qiang LX: Evolution of management for Budd-Chiari syndrome: A team's view from 2564 patients. ANZ J Surg 75: 55-63, 2005.

12. Qi X, Zhang C, Han G, Zhang W, He C, Yin Z, Liu Z, Bai W, Li R, Bai M, et al: Prevalence of the JAK2V617F mutation in Chinese patients with Budd-Chiari syndrome and portal vein thrombosis: A prospective study. J Gastroenterol Hepatol 27: 1036-1043, 2012

13. Wang H, Sun G, Zhang P, Zhang J, Gui E, Zu M, Jia E, Xu H, Xu L, Zhang J and Lu Z: JAK2 V617F mutation and 46/1 haplotype in Chinese Budd-Chiari syndrome patients. J Gastroenterol Hepatol 29: 208-214, 2014

14. Valla DC: Hepatic venous outflow tract obstruction etiopathogenesis: Asia versus the West. J Gastroenterol Hepatol 19: S204-S211, 2004.

15. Bartel DP: MicroRNAs: Target recognition and regulatory functions. Cell 136: 215-233, 2009.

16. Bartel DP: MicroRNAs: Genomics, biogenesis, mechanism and function. Cell 116: 281-297, 2004.

17. Urbich C, Kuehbacher A and Dimmeler S: Role of microRNAs in vascular diseases, inflammation and angiogenesis. Cardiovasc Res 79: 581-588, 2008.

18. Small EM and Olson EN: Pervasive roles of microRNAs in cardiovascular biology. Nature 469: 336-342, 2011.

19. Yue J, Guan J, Wang X, Zhang L, Yang Z, Ao Q, Deng Y, Zhu P and Wang G: MicroRNA-206 is involved in hypoxia-induced pulmonary hypertension through targeting of the HIF-1 $\alpha /$ Fhl-1 pathway. Lab Invest 93: 748-759, 2013.

20. Icli B, Wara AK, Moslehi J, Sun X, Plovie E, Cahill M, Marchini JF, Schissler A, Padera RF, Shi J, et al: MicroRNA-26a regulates pathological and physiological angiogenesis by targeting BMP/SMAD1 signaling. Circ Res 113: 1231-1241, 2013

21. Zhao Z, Zhao Q, Warrick J, Lockwood CM, Woodworth A, Moley KH and Gronowski AM: Circulating microRNA miR-323-3p as a biomarker of ectopic pregnancy. Clin Chem 58: 896-905, 2012

22. Tan L, Yu JT, Liu QY, Tan MS, Zhang W, Hu N, Wang YL, Sun L and Jiang T: Circulating miR-125b as a biomarker of Alzheimer's disease. J Neurol Sci 336: 52-56, 2014.

23. El-Hefnawy T, Raja S, Kelly L, Bigbee WL, Kirkwood JM, Luketich JD and Godfrey TE: Characterization of amplifiable, circulating RNA in plasma and its potential as a tool for cancer diagnostics. Clin Chem 50: 564-573, 2004.

24. Mitchell PS, Parkin RK, Kroh EM, Fritz BR, Wyman SK Pogosova-Agadjanyan EL, Peterson A, Noteboom J, O'Briant KC, Allen A, et al: Circulating microRNAs as stable blood-based markers for cancer detection. Proc Natl Acad Sci USA 105 10513-10518, 2008.

25. Ach RA, Wang H and Curry B: Measuring microRNAs: Comparisons of microarray and quantitative PCR measurements, and of different total RNA prep methods. BMC Biotechno 8: 69, 2008.

26. Kroh EM, Parkin RK, Mitchell PS and Tewari M: Analysis of circulating microRNA biomarkers in plasma and serum using quantitative reverse transcription-PCR (qRT-PCR). Methods 50: 298-301, 2010

27. Livak KJ and Schmittgen TD: Analysis of relative gene expression data using real-time quantitative PCR and the 2(-Delta Delta C(T)) Method. Methods 25: 402-408, 2001.

28. Li D, Yang P, Xiong Q, Song X, Yang X, Liu L, Yuan W and Rui YC: MicroRNA-125a/b-5p inhibits endothelin-1 expression in vascular endothelial cells. J Hypertens 28: 1646-1654, 2010.

29. Wang E, Nie Y, Zhao Q, Wang W, Huang J, Liao Z, Zhang H, $\mathrm{Hu} \mathrm{S}$ and Zheng Z: Circulating miRNAs reflect early myocardial injury and recovery after heart transplantation. J Cardiothorac Surg 8: 165, 2013

30. Tijsen AJ, Creemers EE, Moerland PD, de Windt LJ, van der Wal AC, Kok WE and Pinto YM: MiR423-5p as a circulating biomarker for heart failure. Circ Res 106: 1035-1039, 2010.
31. Chen L, Lu MH, Zhang D, Hao NB, Fan YH, Wu YY, Wang SM, Xie R, Fang DC, Zhang H, et al: miR-1207-5p and miR-1266 suppress gastric cancer growth and invasion by targeting telomerase reverse transcriptase. Cell Death Dis 5: e1034, 2014.

32. Jia L, Wu J, Zhang L Chen J, Zhong D, Xu S, Xie C and Cai J: Restoration of miR-1228* expression suppresses epithelial-mesenchymal transition in gastric cancer. PLoS One 8: e58637, 2013

33. Fu Y, Yi Z, Wu X, Li J and Xu F: Circulating microRNAs in patients with active pulmonary tuberculosis. J Clin Microbiol 49: 4246-4251, 2011

34. Li D, Yang P, Xiong Q, Song X, Yang X, Liu L, Yuan W and Rui YC: MicroRNA-125a/b-5p inhibits endothelin-1 expression in vascular endothelial cells. J Hypertens 28: 1646-1654, 2010.

35. Reijerkerk A, Lopez-Ramirez MA, van Het Hof B, Drexhage JA, Kamphuis WW, Kooij G, Vos JB, van der Pouw Kraan TC, van Zonneveld AJ, Horrevoets AJ, et al: MicroRNAs regulate human brain endothelial cell-barrier function in inflammation: Implications for multiple sclerosis. J Neurosci 33: 6857-6863, 2013.

36. Che P, Liu J, Shan Z, Wu R, Yao C, Cui J, Zhu X, Wang J, Burnett MS, Wang S and Wang J: MiR-125a-5p impairs endothelial cell angiogenesis in aging mice via RTEF-1 downregulation. Aging Cell 13: 926-934, 2014.

37. Hao L, Wang XG, Cheng JD, You SZ, Ma SH, Zhong X, Quan L and Luo B: The up-regulation of endothelin-1 and down-regulation of miRNA-125a-5p, -155 and $-199 a / b-3 p$ in human atherosclerotic coronary artery. Cardiovasc Pathol 23: 217-223, 2014.

38. Dashwood MR and Tsui JC: Endothelin-1 and atherosclerosis: Potential complications associated with endothelin-receptor blockade. Atherosclerosis 160: 297-304, 2002.

39. Kowala MC: The role of endothelin in the pathogenesis of atherosclerosis. Adv Pharmacol 37: 299-318, 1997.

40. Zhao H, Li M, Li L, Yang X, Lan G and Zhang Y: MiR-133b is down-regulated in human osteosarcoma and inhibits osteosarcoma cells proliferation, migration and invasion and promotes apoptosis. PLoS One 8: e83571, 2013.

41. Zhao Y, Huang J, Zhang L, Qu Y, Li J, Yu B, Yan M, Yu Y, Liu B and Zhu Z: MiR-133b is frequently decreased in gastric cancer and its overexpression reduces the metastatic potential of gastric cancer cells. BMC Cancer 14: 34, 2014.

42. Wang E, Nie Y, Zhao Q, Wang W, Huang J, Liao Z, Zhang H, $\mathrm{Hu}$ S and Zheng Z: Circulating miRNAs reflect early myocardial injury and recovery after heart transplantation. J Cardiothorac Surg 8: 165, 2013.

43. Tijsen AJ, Creemers EE, Moerland PD, de Windt LJ, van der Wal AC, Kok WE and Pinto YM: MiR423-5p as a circulating biomarker for heart failure. Circ Res 106: 1035-1039, 2010.

44. Chen L, Lü MH, Zhang D, Hao NB, Fan YH, Wu YY, Wang SM Xie R, Fang DC, Zhang H, et al: MiR-1207-5p and miR-1266 suppress gastric cancer growth and invasion by targeting telomerase reverse transcriptase. Cell Death Dis 5: e1034, 2014.

45. Jia L, Wu J, Zhang L, Chen J, Zhong D, Xu S, Xie C and Cai J: Restoration of miR-1228* expression suppresses epithelial-mesenchymal transition in gastric cancer. PLoS One 8: e58637, 2013.

46. Pentassuglia L and Sawyer DB: ErbB/integrin signaling interactions in regulation of myocardial cell-cell and cell-matrix interactions. Biochim Biophys Acta 1833: 909-916, 2013.

47. Koziński K and Dobrzyń A: Wnt signaling pathway-its role in regulation of cell metabolism. Postepy Hig Med Dosw (Online) 67: 1098-1108, 2013.

48. Siddiqui SS, Siddiqui ZK, Uddin S, Minshall RD and Malik AB: P38 MAPK activation coupled to endocytosis is a determinant of endothelial monolayer integrity. Am J Physiol Lung Cell Mol Physiol 292: L114-L124, 2007.

49. Liang $X, X u$ F, Li X, Ma C, Zhang Y and Xu W: VEGF signal system: The application of antiangiogenesis. Curr Med Chem 21: 894-910, 2014.

50. Shibuya M: Vascular endothelial growth factor and its receptor system: Physiological functions in angiogenesis and pathological roles in various diseases. J Biochem 153: 13-19, 2013. 